\title{
IOT Based Smart Monitoring System for Fish Farming
}

\author{
A.Ramya, R.Rohini, S.Ravi
}

\begin{abstract}
The purpose of the current method is to create a safe and secure that helps the fish pond owners and aquatic planters in producing high quality fish by maintaining normal water levels in the fish tank. The flow of the low or high water in the fish pond will solve the long-term problem of killing fish in a fish tank. Each water quality can affect the health of animals alone. The flow of water on fish ponds discusses how every day should be monitored. This should ensure quality by handling the $\mathrm{PH}$, membrane, temperature, ammonia etc. It is a symbol of good quality water quality standards and poor water quality pools and how it should be upgraded. It is recommended that a prerequisite to increase production by ensuring sustainable fresh quality, and consequently, priority should be given priority. Therefore, water quality parameters maintain balanced positions, culture is the basis for the health and development of living organisms. It is recommended to monitor and evaluate water quality parameters on a regular basis
\end{abstract}

Keywords - Fish pond, Microcontroller,pH sensor,IOT.

\section{INTRODUCTION}

This area reconsider some of the already existing automatic fish feed systems. Most automated fish feeding tools are not easy to control the size of the published food. For an indoor fish feeding system such as water tank, a small device may be appropriate but external pools may need to create a larger system for saving large water. Determines the number of fish stockpiles of fish size of fish size and the fish of the dense aquarium fish. The method is to monitor and control the fish tank through the Internet. The level of fish and the quantity of food items can be properly monitored. The purpose of this system is to design and develop a fish feed system. The fish owner will be able to personalize the feeding time or to feed their fish immediately without delay. Fish owner can monitor the environment of the tank using the Internet and check the food level. The purpose and definition of this system are listed below according to identification and usage objectives and this system can only dissolve dry food. This method is used online and can monitor the fish tank environment and check the status of the previous meal.

\section{REALTED WORKS}

Fish are generally not able to feed at fish when they are usually unable to recover when the fish are usually away from home environment [2]. Two reasons, the risk of

Revised Manuscript Received on August 14, 2019.

A.Ramya, P.G. Scholar Department, of Computer Science and Engineering, Vivekanandha College of Engineering for women, Tamilnadu, India. (E-mail: ramrajasanthi37@gmail.com)

R.Rohini, Associate Professor, Department of Computer Science and Engineering, Vivekanandha College of Engineering for Women, Tamilnadu, India. (E-mail: rohinir84@gmail.com)

S.Ravi, Senior Lecturer, Botswana International University of Science and Technology, Botswana. (E-mail: drravieee@gmail.com) feeding starvation and fish, leads to poor water quality in fish wells and indoor fish pots. It is therefore important to monitor fish fasting, which is very useful for fish proprietors. The work is planned for assuaging fish [4] to reestablish fish at the hour of encouraging their fish utilizing a fish-bolstered mode and microcontroller and raspberry pack based web application. . In this structure, fish proprietor can screen a fish tank for appropriate activity of the fish proprietor [4]. Moreover, the client can set fish feed tables through the web application. This strategy likewise can record valuable sustenance timing, and the client can see them from the UI [5]. The interface can be gotten to through the associated site utilizing raspberry pie. Other usefulness on the interface incorporates a fish feed catch naturally, changing or changing the nourishment setting lastly camera work. Associating the angler's Internet association can utilize the camera appended to the tanker to check the mass of the tank. This is to guarantee that the client isn't going [6].

\section{EXISTING SYSTEM}

\section{Fish feeder system using Raspberry Pi}

In the existing method we used Internet of thing (IOT) using Raspberry Pi. The individuals having local pet fish are in trouble when they aren't around the local area to nourish and deal with their fish. This may prompt overloading or starvation of the fish without feed. Right now there are Arduino based frameworks that could fill the need with planned sustaining of the fish. In any case, the proprietors will be still in trouble for not knowing the state of the fish from their remote areas. The mechanical part comprises of the stepper engine constrained by the Raspberry Pi B+ through web interface, which can apportion sustenance pellets by means of suitable revolutions of the compartment containing the nourishment pellets according to the overseer's inclination. The electrical part comprises of a Raspberry Pi B+ module and a pi-camera, which gives web interface and the continuous video information accumulation of fish. The web interface comprises of the client fixed booking, bolstering information, live spilling of fish, and so on. In the manual nourishing, the client must sustain the fish remotely through the web interface. So, the module can be utilized for planned sustaining of the fish or remotely feed through web interface.

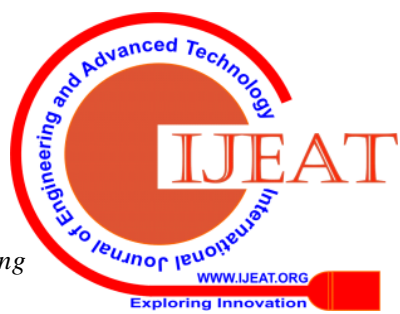




\section{IOT BASED SMART MONITORING SYSTEM FOR FISH FARMING}

\section{PROPOSED SYSTEM \& RESULTS}

\section{A) Fish pond maintained using controller}

In this proposed system of microcontroller based fish pond maintained using the sensors. The ultrasonic and $\mathrm{PH}$ sensors are fixed in the pond system to monitor the water level and water quality. The ultrasonic sensors are used to sense the object of the fish living the pond, and the pulse to send and receive the echo of the sound in the system of pond system. The PH sensor is used to sense in level of water in a pond system. The microcontroller are used to control in a whole circuit of the system, LCD display are connected to microcontroller with any fault or issues will indicate to display the problem. The method discusses but water stream into fish lakes got the opportunity to be observed every day and it quality guaranteed through the control of parameters like: hydrogen particle fixation, turbidity, temperature, smelling salts, and so forth.

It any discussesthe lists of fine quality water and accordingly the impacts of poor water quality in lakes and the way it should be improved upon.It suggests that guaranteeing feasible great water quality is a precondition for expanded profitability and the other way around and ought to in this manner be given direct need Thus, keeping up adjusted degrees of water quality parameters is prime for each the wellbeing and development of culture living beings.

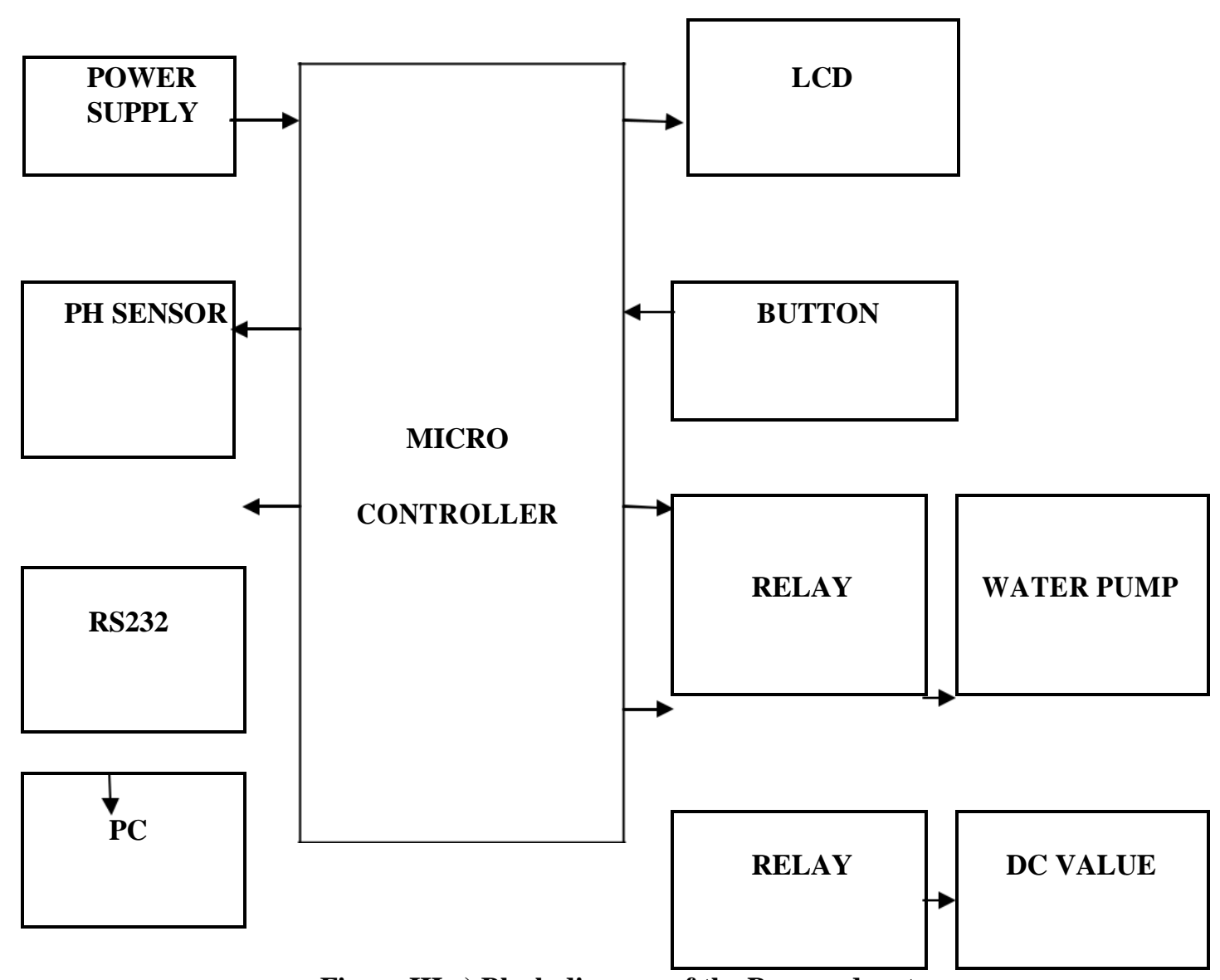

Figure III.a) Block diagram of the Proposed system

\section{B. Construction.}

* To measure the status of the water, pH sensor, humidity sensor, water level sensor and dissolved oxygen sensor had been used. The $\mathrm{pH}$ sensor measures the acidic or basic nature of the water in the pond

* The ultrasonic sensor is find the obstacle in the water tank

* The power supply gives the input supply interface to the microcontroller

* The RS 232 is interface both hardware andsoftware

* The power supply circuit consists of step down transformer which is $230 \mathrm{v}$ step down to $12 \mathrm{v}$.In this circuit 4diodes are used to form bridge rectifier which delivers pulsating dc voltage \& then fed to condenser filter the output voltage from rectifier is fed to filter.

* The filtered DC voltage is given to regulator to supply $12 \mathrm{v}$ constant DC voltage.

* LCD Liquid crystal display are interfacing to microcontroller. This 5V DC is used to supply power to the controller and the LCD. Power supply to the LCD is given from the voltage regulator.

* A transformer is AN electrical regulator designed to mechanically maintain a relentless voltage level.

* In this project, power offer of $5 \mathrm{~V}$ and $12 \mathrm{~V}$ area unit needed 


\section{Hardware Explanation}

i) Ph sensor

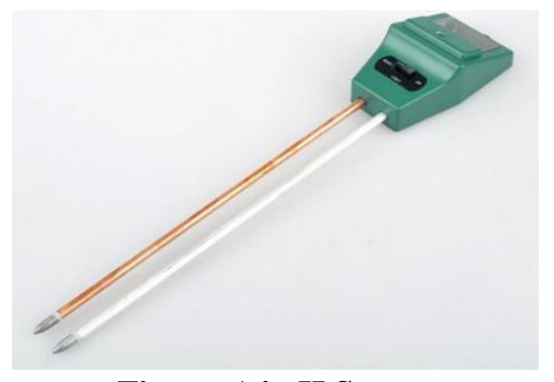

Figure c).i pH Sensor

A $\mathrm{pH}$ meter is a logical instrument estimating hydrogen-particle work in water-based arrangements, its causticity or alkalinity. $\mathrm{pH}$ meter estimates the distinction in power between a $\mathrm{PHH}$ and one reference voltage, so the $\mathrm{pH}$ meter is here and there alluded to as a "metric $\mathrm{pH}$ meter". The difference in electric energy is related to the acidity of the solution or the $\mathrm{pH}$. The $\mathrm{pH}$ meter is used in various applications ranging from laboratory tests to quality control.

\section{ii) Ultrasonic sensor}

The ultrasonic sensor is a 4 stick hinder, whose stick names are Vcc, Trigger, Echo and Ground individually. This sensor is a famous sensor utilized in numerous applications, where separation or sensitivity is needed. The block consists of two eyes, such as an ultrasonic transmitter and pre-programmable recipients. The sensor ultrasonic transmitter works with a simple high school formula that passes an ultrasonic wave, and this reflected wave ultrasonic receiver block is observed when the wave travels the air and it senses the reaction when it opposes any substance



Figure c).ii Ultrasonic Sensor

\section{iii) PIC microcontroller}

PIC Microcontroller Pic16f877a is one of the triumphs control in the strategy. This controller is exceptionally advantageous to utilize, this controller is all the more simple to record or programming. One of the real favorable circumstances is that it can compose and delete ordinarily by utilizing streak memory innovation. This is the complete number of 40 pins and the info and yield are 33 pins. PIC16f877a is utilized in numerous PIC microcontroller programs. Pic16f877a Digital Electronics Circuit has numerous applications. Pic16f877a detects its applications in a large number of devicesPIC16F877A Introduction.
Working Principal Of LCD

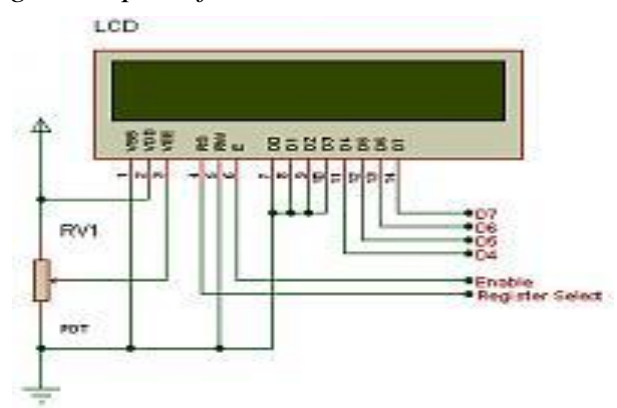

Figure c).iii Simulation Diagram of LCD

The standard behind the LCD's is that once partner electrical flow is connected to the fluid particle, the atom will in general fix out.This causes the edge of light which is going through the atom of the enraptured glass and furthermore cause an adjustment in the point of the top polarizing filter.As an outcome a touch lightweight is permitted to pass the captivated glass through a chose space of the alphanumeric display.Thus that definite space can wind up dim contrasted with alternative. The alphanumeric showcase chips away at the guideline of square lightweight. While building the LCD's, a reflected mirror is composed at the rear.An conveyor plane is framed of indium-tin synthetic intensify that is whole on high and a spellbound glass with a polarizing film.

\section{iv) Interfacing Lcd With Microcontroller}

* Interfacing LCD with microcontroller is extremely simple errand if the best possible LCD programming calculation is known.

* LCD utilized here has HD44780u spot framework LCD controller.

* LCD module has 8-piece information interface and the executives pins.One will send learning as 8piece or in consolidate of 24 -piece snack.

\section{LCD Initialization}

* Steps to introduce the LCD

* Specify capacity set: Send $38 \mathrm{H}$ for 8-piece, twofold line and 5x7 spot character group.

* Display On-Off control: Send 0FH for showcase and squint cursor on.

* Entry mode set: Send $06 \mathrm{H}$ for cursor in augmentation position and move is undetectable.

* Clear show: Send 01H to clear show and return cursor to home position

* Next venture after instatement is to send information bytes to required showcase information RAM memory

* Location. Right off the bat set the location area utilizing address set direction byte and after that send information bytes utilizing

* The DDRAM compose direction. To address explicit area in showcase information RAM one must have the learning of how the location counter is increased. 


\section{IOT BASED SMART MONITORING SYSTEM FOR FISH FARMING}

$16 X 2 L C D$

- 16X2 LCD can be used to display 16 characters in 2 rows. It has the ability to display numbers,

* Characters and graphics. It has an inbuilt refreshing circuit, thereby relieving the CPU from the task of

* Refreshing. LCD discussed has total of 14 pins

RS 232

In this strategy to correspondences between PC hardware over phone lines, RS232 is presently generally utilized for direct associations between information obtaining gadgets and PC frameworks. As inside the meaning of RS232, the PC is data transmission equipment (DTE).

Be that as it may, a few interface item don't appear to be information interchanges instrumentalty (DCE) .Null electronic hardware links territory unit intended for this circumstance; rather than having the stick high in associations of electronic gear links, invalid electronic hardware links have totally unique inward wiring to allow DTE gadgets to speak with each other. RS-232 links region unit unremarkably out there with either four, nine or 25 -stick wiring. The 25 -stick link interfaces each stick; the 9-stick links don't encapsulate a few of the exceptionally utilized associations; 4-stick links offer the empty least associations, and have jumpers to give "handshaking" for those gadgets that require it. These jumpers interface pins four, 5 and 8 , and furthermore sticks 6 and 20.The approach of the IBM PC AT has made a fresh out of the plastic new wrinkle in RS232 correspondences.

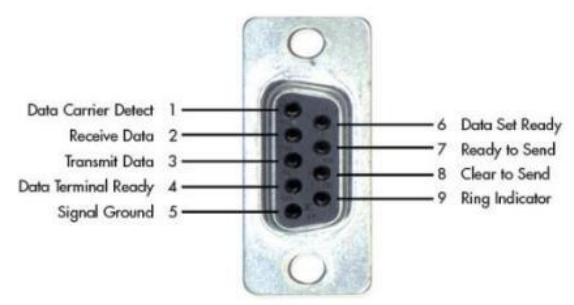

Figure c).iv RS 232 Specfication

\section{v) RELAY}

We realize that most of the high complete modern application gadgets have transfers for their successful operating.Relays square measure straight forward switches that square measure worked each electrically and naturally. Transfers contains partner degree magnet and conjointly a gathering of contacts. The exchanging component is completed with the assistance of the electromagnet. There additionally are elective in activity standards for its working. Be that as it may, they contrast as per their applications. A large portion of the gadgets have the applying of transfers.

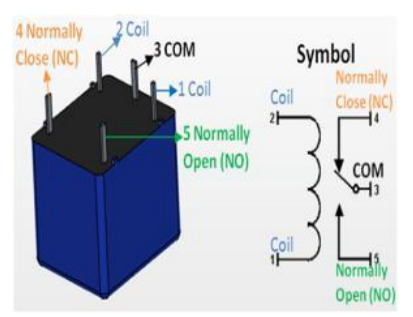

Figure c).v Circuit diagram of Relay

\section{CONCLUSION}

The microcontroller based self-kept up aquarium utilizing with sensors framework utilizes the utilization of various advances in its structure, improvement, and execution. The framework utilized microcontroller to screen the procedure of fish tank in an over-head tank stockpiling framework and can identify the degree of water in a tank, switch on/off the tank utilizing sensors as needs be and show the status on a LCD screen. This strategy has effectively given an enhancement for existing water level controllers.

\section{FUTURE SCOPE}

The degree behind building up the programmed fish bolstering framework is to lessen the manual fish sustaining framework which uses more work powers. Also, there are sure preferences that lead to its advancement which are the measure of sustenance that will be conveyed to the water body that will measure or controlled keeping aquarium clean and fishes healthy.Feeders can sustain the fishes notwithstanding when the proprietor is away in a helpful manner. In view of the abilities and functionalities, general battery worked fish feeder gadgets accessible in the market can just suit nourishment like beds, chips and powder, so by including extra compartment for pleasing characteristic fish sustenance's like worms, water escapes and so forth makes the gadget progressively helpful as it were.

\section{REFERENCES}

1. S.Kayalvizhi,Koushik Reddy G, Vivek Kumar P, VenkataPrasanth N (2015)“Cyber Aqua Culture Monitoring System Using Ardunio And Raspberry Pi”, IJAREEIE(international journal of advanced research in electrical, electronics, and instrumentation engineering), Vol: 4, Issue No: 5, pp.no:4554 - 4558.

2. E. N. Onwuka, Achonu O. Adejo and Ibrahim U. Joseph (2011), "Design and Construction of a Microcontrollerbased Automatic Fish Feeding Device", the 26th Annual Conference \& fair of the Fisheries Society of Nigeria, pp.no: $11-15$.

3. Mohd Nor Azni, M.N.H, Vellasami. L, Zianal. A.H, Mohammed.F.A, Mohd Daud.N.N, Vejasegaran.R, N.W.Basharudin, Jusoh.M, $\mathrm{Ku}$ Azir.N.F, P.L.Eh Kan,(2016) "Home Automation System with Android Application",3rd International Conference on Electronic Design (ICED), pp.no:299-303. 
4. Ricardo Yauri Rodriguez, Milton Rios Julcapoma, Rubén Acosta Jacinto, (2016) "Network monitoring environmental quality in agriculture in agriculture and pisciculture with low power sensor node based on zigbee and GPRS technology", XXIII international congress on electronics, electrical engg. and computing (INTERCON), pp.no:1-6.

5. Liu Xingqiao, Geng Jiao, Ji Feng, Zhao Dean, (2008) "Using MATLAB Image Processing to Monitor the Health of Fish in Aquiculture", 27th Chinese Control international Conference proposed in IEEE, pp.no: 677 - 680.

6. Hidayatul Nur Binti Hasim, Mritha Ramalingam, Ferda Ernawan, Puviarasi .R.(2017) "Developing fish feeder system using Raspberry Pi", IEEE 3rd International Conference on Advances in Electrical, Electronics, Information, Communication and BioInformatics (AEEICB17), pp.no: 246 - 250.

7. Jui-Ho Chen, Wen-Tsai Sung and Guo-Yan Lin,(2016) "Automated Monitoring System for the Fish Farm Aquaculture Environment" IEEE International Conference on Systems, Man, and Cybernetics, Vol No.57, Sec. 2,pp.no:1161-1166.

8. M. Z. H. Noor, A. K. Hussian, M. F. Saaid, M. S. A. M. Ali, M. Zolkapli,(2012) "The Design and Development Of Automatic Fish Feeder System Using

9. C.T. Chiang, Y.K. Lu and L.T. Lin, "A CMOS Fish Spoilage Detector for IoT Applications of Fish Markets", IEEE Sensors Journal, Vol. 18, No. 1, Pp. 375-381, 2018.

10. Y. Atoum, S. Srivastava and X. Liu, "Automatic feeding control for dense aquaculture fish tanks", IEEE Signal Processing Letters, Vol. 22, No. 8, Pp. 1089-1093, 2015.

11. B.K. Biswas, Y.M. Svirezhev and B.K. Bala, "A model to predict climate-change impact on fish catch in the world oceans", IEEE Transactions on Systems, Man, and Cybernetics-Part A: Systems and Humans, Vol. 35, No. 6, Pp. 773-783, 2005. 